\title{
Oscillatory Stability Prediction Using PSO Based Synchronizing and Damping Torque Coefficients
}

\author{
N. A. M. Kamari ${ }^{1}$, I. Musirin ${ }^{2}$, Z. A. Hamid ${ }^{3}$, M. H. M. Zaman ${ }^{4}$ \\ ${ }^{1,4}$ Centre for Integrated Systems Engineering and Advanced Technologies (INTEGRA), \\ Faculty of Engineering and Built Environment, National University of Malaysia, Bangi, Selangor, 43600, Malaysia \\ ${ }^{2,3}$ Faculty of Electrical Engineering, Universiti Teknologi Mara, Shah Alam, Selangor, 40450, Malaysia
}

\section{Article Info}

Article history:

Received March 07, 2018

Revised Jul 23, 2018

Accepted Aug 06, 2018

\section{Keywords:}

Damping torque coefficient Minimum damping ratio Particle Swarm Optimization Synchronizing torque coefficient

\begin{abstract}
This paper presents the assessment of stability domains for the angle stability condition of the power system using Particle Swarm Optimization (PSO) technique. An efficient optimization method using PSO for synchronizing torque coefficients $K_{s}$ and damping torque coefficients $K_{d}$ to identify the angle stability condition on multi-machine system. In order to accelerate the determination of angle stability, PSO is proposed to be implemented in this study. The application of the proposed algorithm has been justified as the most accurate with lower computation time as compared to other optimization techniques such as Evolutionary Programming (EP) and Artificial Immune System (AIS). Validation with respect to eigenvalues determination, Least Square (LS) method and minimum damping ratio $\xi_{\text {min }}$ confirmed that the proposed technique is feasible to solve the angle stability problems.
\end{abstract}

Copyright () 2018 Institute of Advanced Engineering and Science. All rights reserved.

\section{Corresponding Author:}

N. A. M. Kamari,

Centre for Integrated Systems Engineering and Advanced Technologies (INTEGRA),

Faculty of Engineering and Built Environment,

National University of Malaysia, Bangi, Selangor, 43600, Malaysia.

Email: azwank@ukm.edu.my

\section{INTRODUCTION}

With the increase of energy consumption in this age, a study on the stability of the power system becomes a necessity, especially small signal stability analysis of power systems. This analysis is used to predict electromagnetic swing at low frequencies, as a result of undisturbed rotor swing. References [1]-[9] have pointed out that the stability of the oscillation in the power system is a very important issue. As the power system operation changes over time, the stability of the small signal in this power system should be tracked online. To track the system, selected stability indicators are calculated from the data provided over time. These indicators are updated until a constant value is obtained. In this paper, synchronizing torque coefficient, $K_{s}$ and damping torque coefficient, $K_{d}$ are used as stability indicators. For a system to achieve a stable condition, both $K_{s}$ and $K_{d}$ values must be positive [5]-[8].

The Least Square method (LS) is one of the techniques in finding this $K_{s}$ and $K_{d}$ value, which has been used as a static parameter estimator [6]-[8]. However, long calculation times and data updating requirements are the weakness in the LS method. This method also requires monitoring throughout the duration of the swing.

Computational intelligence techniques have been widely used in solving power system stability problems. One of them is Evolutionary Programming (EP). This algorithm is heuristic population-based search methods that used both random variation and selection. In this paper, new technique called PSO is proposed. It brought about the performance beyond EP method in searching the optimal solution with faster computation time. 
This paper proposes an efficient technique for estimating synchronizing and damping torque coefficients in solving angle stability problems. This technique is used to estimate the value of $K_{s}$ and $K_{d}$ from the machine responses namely, the change in rotor angle, $\Delta \delta(t)$, the change in rotor speed, $\Delta \omega(t)$ and the change in electromechanical torque, $\Delta T_{e}(t)$. The goal is to minimize the error of the estimated coefficients. The online estimation technique for synchronizing and damping torque coefficients has been tested in IEEE 9-Bus system.

This study presents PSO based technique for angle stability assessment in multi-machine system. Through this study, a mathematical model for multi-machine for the assessment of angle stability is developed. PSO is used to optimize the objective function, $J$ with $K_{s}$ and $K_{d}$ as the control variables. Once the $J$ value has been maximized, $K_{s}$ and $K_{d}$ are analyzed which determines whether the rotor angle is stable or vice versa. The performance of PSO is then compared to EP and AIS. Results obtained from the experiment were then verified with minimum damping ratio, $\xi_{\text {min }}$ and eigenvalues, $\lambda$.

\section{PROBLEM FORMULATION}

To demonstrate the potential of proposed technique in angle stability assessment for multi-machine system, IEEE 9-Bus System model has been selected. Three generators called Generator 1, Generator 2 and Generator 3 are connected to the buses named Bus 1, Bus 2 and Bus 3, respectively. The single line diagram of the test system is showed in Figure 1. The parameters of the system are shown in [9].

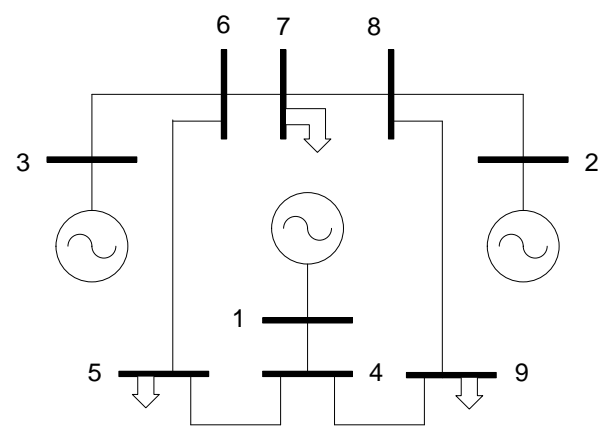

Figure 1. Single line diagram for IEEE 9-bus system

\subsection{Proposed Philips-Heffron Model for Multi-machine System}

A proposed Phillips-Heffron model for the multi machine system is developed and showed in Figure 2. It is developed based on the single machine of Philips-Heffron model [10]. 


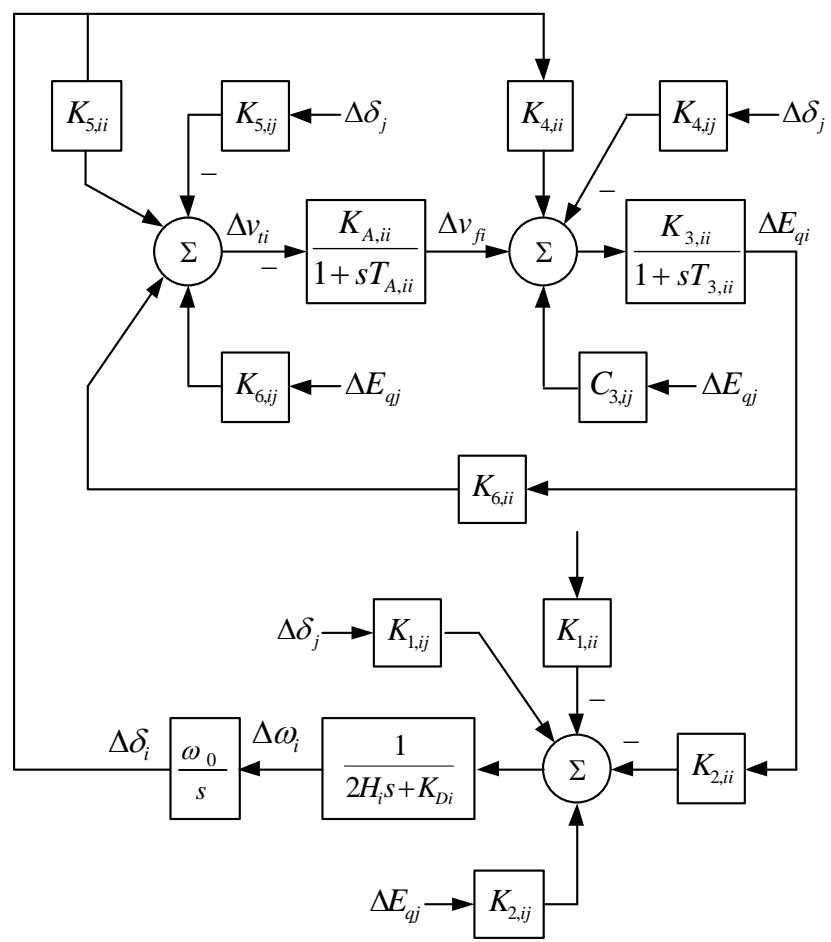

Figure 2. Phillips-Heffron model for multi-machine system

$K_{d}$ is the damping torque coefficient, $H$ is the inertia constant, $K_{A}$ and $T_{A}$ are the circuit constant and time constant of the exciter oscillation system, respectively. $\omega_{0}$ is equal to $2 \pi f_{0} . K_{3}$ is a function of the ratio of impedance. $K_{1}, K_{2}, T_{3}, K_{4}, K_{5}$ and $K_{6}$ are constants which consist of function related to the operating real and reactive loading, electrical torque, rotor speed, rotor angle as well as the excitation levels in the generator.

\subsection{The Developed Mathematical Modelling}

The mathematical modelling can be derived for the proposed Phillips-Heffron model for multi machine system of Figure 2. They are presented in the following mathematical equations:

$$
\begin{aligned}
& \frac{\Delta \omega_{i}}{\Delta t}=\frac{\Delta T_{m i}-\Delta T_{e i}-K_{D i} \Delta \omega_{i}}{2 H_{i}}, i=1, \ldots, m \\
& \frac{\Delta \delta_{i}}{\Delta t}=\omega_{0} \Delta \omega_{i}, i=1, \ldots, m
\end{aligned}
$$

$$
\begin{gathered}
\frac{\Delta E_{q i}}{\Delta t}=\frac{1}{\Delta T_{d 0 i}^{\prime}}\left(\begin{array}{l}
K_{4, i i} \Delta \delta_{i}-\sum_{j \neq i} K_{4, i j} \Delta \delta_{j} \\
-C_{3, i i} \Delta E_{q i}^{\prime}+\sum_{j \neq i} C_{3, i j} \Delta E_{q j}^{\prime} \\
+\Delta v_{f i}
\end{array}\right) \\
i=1, \ldots, m, \quad j=1, \ldots, m, \quad i \neq j
\end{gathered}
$$

$$
\begin{gathered}
\frac{\Delta v_{f i}}{\Delta t}=\frac{K_{A i}}{T_{A i}}\left(\begin{array}{l}
-K_{5, i i} \Delta \delta_{i}+\sum_{j \neq i} K_{5, i j} \Delta \delta_{j} \\
-K_{6, i i} \Delta E_{q i}^{\prime}+\sum_{j \neq i} K_{6, i j} \Delta E_{q j}^{\prime}
\end{array}\right)-\frac{1}{T_{A i}} \Delta v_{f i} \\
i=1, \ldots, m, \quad j=1, \ldots, m, \quad i \neq j
\end{gathered}
$$




$$
\begin{aligned}
\Delta T_{e i} & =K_{1, i i} \Delta \delta_{i}-\sum_{j \neq i} K_{1, i j} \Delta \delta_{j} \\
& +K_{2, i i} \Delta E_{q i}^{\prime}-\sum_{j \neq i} K_{2, i j} \Delta E_{q j}^{\prime} \\
i & =1, \ldots, m, \quad j=1, \ldots, m, \quad i \neq j
\end{aligned}
$$

Details on Equation 1 to 5 are shown in [10]. The Equation 1 to 5 can be rewrite into matrix form as followed:

$$
\begin{aligned}
\dot{X}_{i} & =A_{i} \cdot X_{i}+B_{i} \cdot U_{i}, i=1, \ldots, m \\
X_{i} & =\left[\begin{array}{lll}
\Delta \omega_{r i} \quad \Delta \delta_{i} \quad \Delta E_{q i} & \Delta v_{f i}
\end{array}\right]^{T} \\
i & =1, \ldots, m \\
U_{i} & =\left[\Delta T_{i}\right], i=1, \ldots, m
\end{aligned}
$$

$X_{i}$ and $U_{i}$ are the state vector and input signal vectors for $i$ generators, respectively. The system matrix $A_{i}$ is a function of the system parameters with $i$ generators that depends on the opening conditions. The perturbation matrix $B_{i}$ depends on the system parameters only.

\subsection{Synchronizing and Damping Torque Coefficients}

The correlation between the change in estimated electromagnetic torque deviation, $\Delta T_{e s i}(t)$ with the change in rotor angle, $\Delta \delta_{i}(t)$ and the change in rotor speed, $\Delta \omega_{i}(t)$ for the $i^{\text {th }}$ generator can be expressed as:

$$
\Delta T_{e s i}(t)=K_{s i} \Delta \delta_{i}(t)+K_{d i} \Delta \omega_{i}(t), i=1, \ldots, m
$$

where $K_{s i}$ and $K_{d i}$ are synchronizing torque coefficient and damping torque coefficient for the $i^{\text {th }}$ generator, respectively. $m$ is the number of generators.

The justification of the stability of a linear system can be done by the estimation of $K_{s}$ and $K_{d}$. Positive value of both $K_{s}$ and $K_{d}$ will validate the system as stable. If the system has positive $K_{S}$ and negative $K_{d}$, the system is defined as in oscillatory instability condition. On the other hand, if $K_{s}$ and $K_{d}$ respectively show negative and positive value, the system is considered as non-oscillatory instability condition. In general, the system is said to be unstable if either one of the torque coefficients is negative.

The stability evaluation of a linear system can be predicted by referring to $K_{s}$ and $K_{d}$ values. A stable system is guaranteed if both $K_{s}$ and $K_{d}$ values are positive. Figure 3 illustrated a stable angle stability resulted from a positive value of both $K_{s}$ and $K_{d}[1]$.
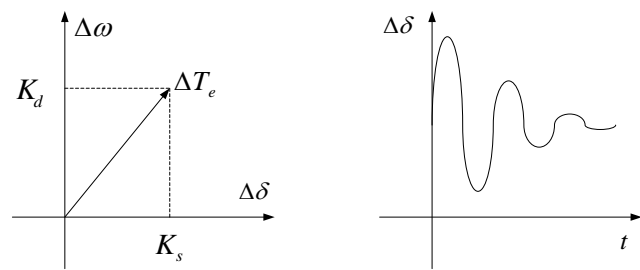

Figure 3. Complex plane of $\Delta T_{e}(t)$ and $\Delta \delta(t)$ response for stable condition

If the linear system has positive $K_{s}$ and negative $K_{d}$, the system is defined as in oscillatory instability condition, which is due to lack of adequate damping torque. The effect of the oscillatory instability condition can be detected from the increment of amplitude oscillations of the rotor. Figure 4 illustrated unstable conditions for angle stability resulted from positive value of $K_{s}$ and negative value of $K_{d}$. 

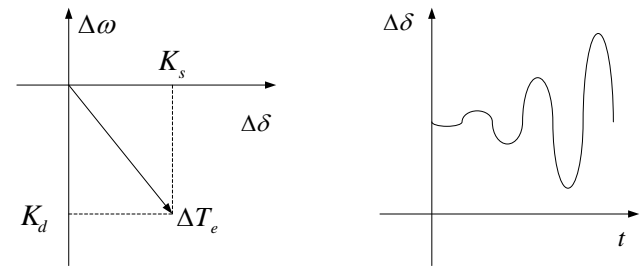

Figure 4. Complex plane of $\Delta T_{e}(t)$ and $\Delta \delta(t)$ response for oscillatory instability condition

Non-oscillatory instability occurred if $K_{s}$ and $K_{d}$, respectively show negative and positive value. This is due to the absence of automatic voltage regulators, resulting lack of sufficient synchronizing torque. This condition can be verified from steady increment of rotor angle response. Figure 5 illustrated unstable conditions for angle stability resulted from negative value of $K_{s}$ and positive value of $K_{d}$.
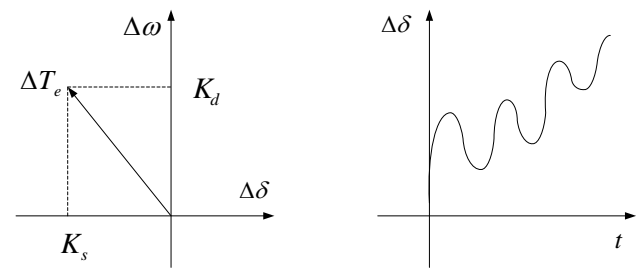

Figure 5. Complex plane of $\Delta T_{e}(t)$ and $\Delta \delta(t)$ response for non-oscillatory instability condition

\subsection{Eigenvalues, Damping Ratio and Damping Factor}

The scalar parameter of eigenvalues, $\lambda$ can be derived as follows [1]:

$$
(A-\lambda \mathrm{I}) \phi=0
$$

where $A$ is an $n \times n$ matrix and $\phi$ is an $n \times 1$ vector.

The $n$ solutions of $\lambda=\lambda_{1}, \lambda_{2}, \ldots, \lambda_{n}$ are eigenvalues of $A$. The $i^{\text {th }}$ eigenvalue can be stated as follows:

$$
\lambda_{i}=\sigma_{i} \pm j \omega_{i}
$$

where $\sigma_{i}$ is the real part of the $i^{\text {th }}$ eigenvalue and $\omega_{i}$ is the imaginary part of the $i^{\text {th }}$ eigenvalue.

The linear system is stable if all eigenvalues have negative real parts. The damping ratio, $\xi_{i}$ for the $i^{\text {th }}$ eigenvalue is defined as:

$$
\xi_{i}=-\sigma_{i} / \sqrt{\sigma_{i}^{2}+\omega_{i}^{2}}
$$

The linear system is certainly in stable condition if all damping ratio have positive value. For simplification purposes, only the minimum value of damping ratio, $\xi_{\min }$ for the linear system is selected to verify the result.

\subsection{Least Square (LS) Method}

Least square (LS) technique is used to minimize the sum of the square of the differences between $\Delta T_{e}(t)$ and $\Delta T_{e s}(t)$. The error is defined as [5], [7]:

$$
E(t)=\Delta T_{e}(t)-\Delta T_{e s}(t)
$$

where $\Delta T_{e}(t)$ : the electrical torque $\Delta T_{e s}(t)$ : the estimated electrical torque.

The value of $t_{\text {total }}$ is as follows: 


$$
t_{\text {total }}=N \times T
$$

where $N$ is the number of samples $T$ is the sampling period.

To estimate the correct value for $K_{s}$ and $K_{d}$, the period of $t_{\text {total }}$ must be chosen. Different value of $t_{\text {total }}$ will result an inaccurate value for $K_{s}$ and $K_{d}$. [7] and [8] have stated that, the suitable value for $t_{\text {total }}$ that makes $K_{s}$ and $K_{d}$ constant during the oscillation period is the value of the entire period of oscillation. In matrix notation, the above problem can be described by an over-determined system of linear equations as follows:

$$
\begin{aligned}
& \Delta T_{e}(t)=\Delta T_{e s}(t)+E(t)=C x+E(t) \\
& C=\left[\begin{array}{ll}
\Delta \delta(t) & \Delta \omega(t)
\end{array}\right] \\
& x=\left[\begin{array}{ll}
K_{s} & K_{d}
\end{array}\right]^{T}
\end{aligned}
$$

where $\Delta T_{e}(t)$ : the electrical torque $\Delta T_{e s}(t)$ : the estimated electrical torque $E(t)$ : differences (error) between $\Delta T_{e}(t)$ and $\Delta T_{e s}(t)$

The estimated vector, $x$ is such that the function, $F(x)$ is minimized, where

$$
F(x)=\left[\Delta T_{e}-A x\right]^{T} \cdot\left[\Delta T_{e}-A x\right]
$$

In this case, $x$ will be given by:

$$
+x=\left[A^{T} \cdot A\right]^{-1} \cdot A^{T} \cdot \Delta T_{e}=A^{t} \cdot \Delta T_{e}
$$

where $A^{t}$ is the left pseudo inverse matrix.

By solving Equation 19, it gives the values of $K_{s}$ and $K_{d}$ for the corresponding operating point. Although the calculated values are accurate, the application of LS method is time consuming and requires the entire period of oscillation [7]-[8]. Due to that, new indicator is needed.

\section{COMPUTATIONAL INTELLIGENCE APPROACHES}

Lately, the use of Artificial Intelligence (AI) technology is synonymous in solving power system problems. AI techniques utilize the logic and knowledge representations of expert systems, artificial neural network (ANN) [11]-[12] and evolutionary computation (EC). The EC field includes PSO [13]-[17], Evolutionary Programming (EP) [8], [18]-[19], Artificial Immune Systems (AIS) [20]-[22] and Firefly Algorithm (FA) [23]-[24]. In this study, EP, PSO and AIS were selected as optimization techniques.

\subsection{Particle Swarm Optimization}

PSO was introduced by Dr. Russ Eberhart and Dr. James Kennedy in 1995. PSO is an evolutionary based optimization technique, which imitates the behaviour of birds flocking and fish schooling. The PSO algorithm is started with initialization, followed by the update of velocity and position, fitness calculation, the best position update and convergence test. The flow chart which represents the PSO algorithm is illustrated in Figure 6. 


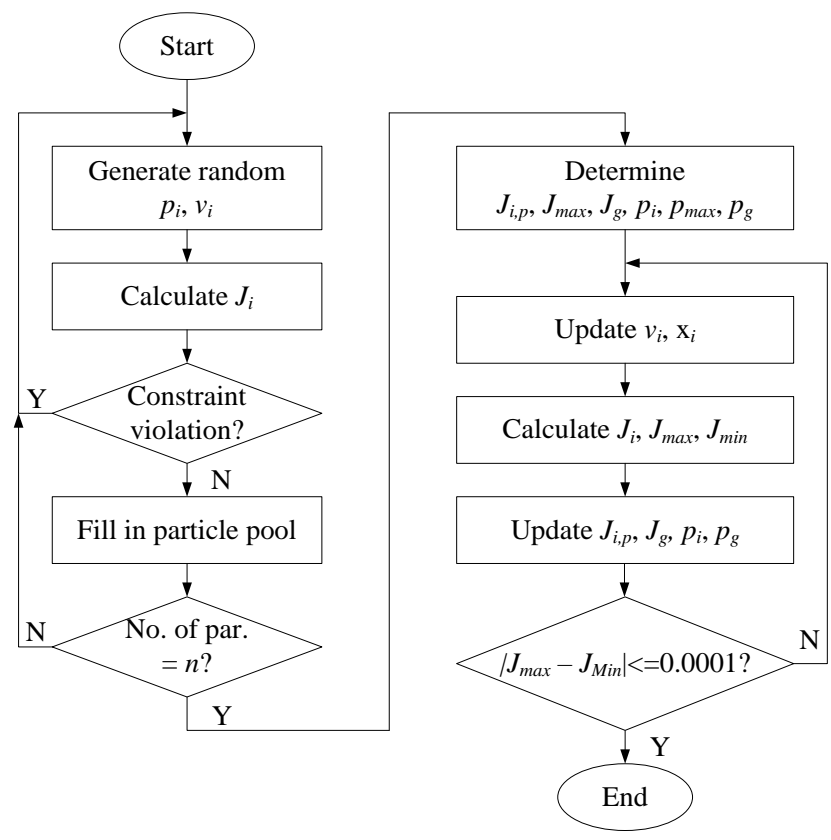

Figure 6. Flowchart for PSO algorithm

Parameters such as acceleration coefficients and inertia weight determine the performance of PSO technique. The inertia weight carries a huge impact on the convergence capability of PSO. A small inertia weight can facilitate a local exploration, while a large inertia weight will facilitate an exploration globally. In this study, the evaluation of inertia weight is performed using Linearly Decreasing Inertia Weight (LDIW) method. In the LDIW approach, the inertia weight, $\omega$ is designed to decrease linearly during the evolution of the PSO, from a maximum value, $\omega_{\max }$, decreases to a minimum value, $\omega_{\min }$ [17]. The equation of inertia weight using LDIW approach is given in:

$$
\omega_{k}=\omega_{\max }-\left(k / \text { iter }_{\max }\right) \cdot\left(\omega_{\max }-\omega_{\min }\right)
$$

where $i t e r_{\max }$ is the maximum number of iteration and $k$ is the current iteration.

\subsection{Evolutionary Programming}

The Evolutionary Programming (EP) uses the models of biological evolutionary process to obtain the solution for complex engineering problems. Invented by D. Fogel in 1962, the optimal solution search method using EP technique is accomplished in a parallel method within the parameter search space. The process of Evolutionary Programming (EP) algorithm includes initialization, statistical evaluation, fitness calculation, mutation, combination and selection. The overall process of EP algorithm is given in [8]

\subsection{Artificial Immune Systems}

Artificial Immune System (AIS) and EP share many common aspects of optimization techniques. EP is based on the natural evolution model, while AIS tries to benefit from the characteristics of a human immune system. AIS involves initialization, statistical evaluation, fitness calculation, cloning, mutation, combination and selection. The whole process is given in [20].

\subsection{Objective Functions}

In this study, the objective function formulated is based on the differences of the electromagnetic torque and the estimated electromagnetic torque of the $i^{\text {th }}$ generator, $\Delta T_{e i}(t)$ and $\Delta T_{e s i}(t)$, respectively, as shown in Equation 21. This difference or error is estimated for calculating $K_{s}$ and $K_{d}$ for every generator in the system. PSO optimization technique is used to minimize the error with $K_{s}$ and $K_{d}$ being the control variables [7]. 


$$
J_{i}=i n v\left(1+\left|\frac{\Delta T_{e i}(t)-\Delta T_{e s i}(t)}{\Delta T_{e i}(t)}\right|\right), i=1, \ldots, m
$$

where $m$ is the number of generators. Hence, the objective function can be defined as:

$\operatorname{Maximize}\left(J_{i}\right)$

From the optimized $J$ value, decision can be made to identify the angle stability based on the values of $K_{s}$ and $K_{d}$.

\subsection{Algorithm for Angle Stability Assessment}

To estimate successfully the maximum value of $J_{i}$, the calculation process of $K_{s i}$ and $K_{d i}$ for the $i^{\text {th }}$ generator is conducted repeatedly. The following process is implemented:

a. Calculate $\Delta T_{e s i}(t)$ using $\Delta \delta_{i}(t), \Delta \omega_{i}(t)$ and the estimated torque coefficients using Equation 9.

b. Evaluate $J_{i}$ using Equation 21 .

c. If $J_{i}$ is smaller than 1.00 , vary the value of $K_{s i}$ and $K_{d i}$ and repeat step (i) and (ii) with new generated $\Delta \delta_{i}(t)$ and $\Delta \omega_{i}(t)$ sample data until $J_{i}$ reaches 1.00 or all sample data were used.

The process is simplified in the form of a flowchart, as shown in Figure 7.

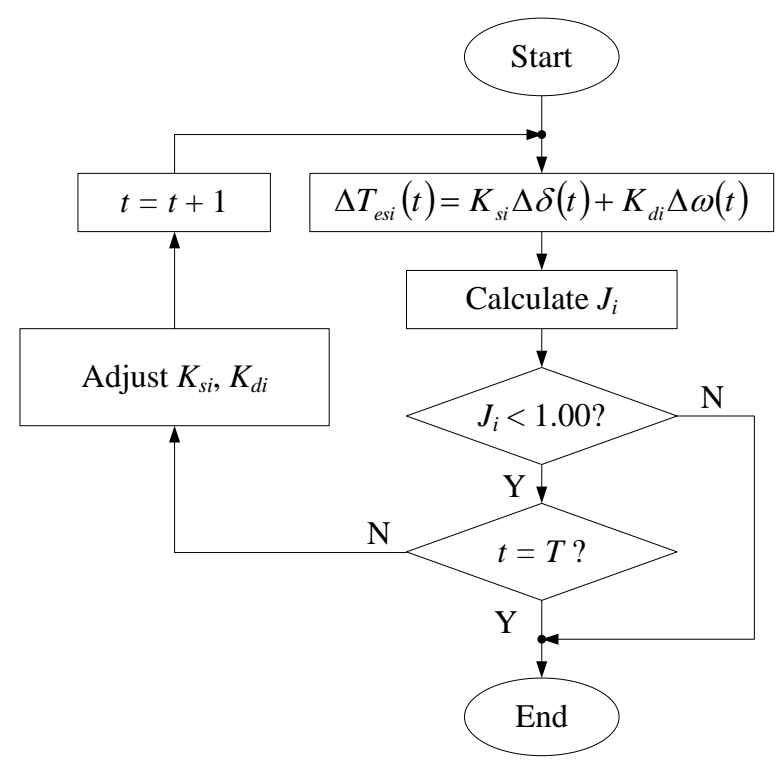

Figure 7. Flowchart for $\mathrm{K}_{\mathrm{s}}$ and $\mathrm{K}_{\mathrm{d}}$ estimation process

Table 1 tabulates the parameters used in PSO optimization process. There are four parameters needed for the PSO as the optimization technique i.e. $c_{l}$ and $c_{2}$ are acceleration coefficients, $\omega_{\min }$ and $\omega_{\max }$ are the minimum inertia weight and the maximum inertia weight, respectively.

Table 1. Parameters of EP, AIS and PSO

\begin{tabular}{cccc}
\hline Parameters & EP & AIS & PSO \\
\hline$c_{l}$ and $c_{2}$ & - & - & 0.9 \\
$\omega_{\min }$ & - & - & 0.04 \\
$\omega_{\max }$ & - & - & 0.09 \\
$\beta$ & 0.05 & 0.05 & - \\
Population Size & 20 & 20 & 20 \\
\hline
\end{tabular}




\section{RESULTS AND DISCUSSION}

In this study, the performance of PSO technique in estimating $K_{s}$ and $K_{d}$ are conducted on IEEE 9bus system. Three samples of data of rotor angle, $\Delta \delta(t)$, rotor speed, $\Delta \omega(t)$ and electrical torque, $\Delta T_{e}(t)$ for all three generators are produced in Matlab Simulink environment. To simulate various stability cases, three different value of reactive load at Bus 5 is used. The values of the reactive load at Bus 5 are chosen in such a way that three scenarios can be emulated, namely unstable, critically stable and stable conditions as tabulated in Table 2.

Table 2. Three Different Loading Conditions

\begin{tabular}{lc}
\hline \multicolumn{1}{c}{ Cases } & Reactive Load at Bus 5 \\
\hline Case 1 (unstable condition) & $160 \mathrm{MVar}$ \\
Case 2 (critically stable condition) & $60 \mathrm{MVar}$ \\
Case 3 (stable condition) & $10 \mathrm{MVar}$ \\
\hline
\end{tabular}

Responses of $\Delta \delta(t), \Delta \omega(t)$ and $\Delta T_{e}(t)$ for Cases 1, 2 and 3 are shown in Figure 8, Figure 9 and Figure 10 , respectively. To evaluate the capability of the proposed scheme, the minimum damping ratio $\xi_{\min }$ and eigenvalues $\lambda$ are calculated and were set as a benchmark. In stable condition, $\delta_{\min }$ will give a positive result meanwhile all values of $\lambda$ will show negative values. On the contrary, if the system is unstable, it will result to negative $\xi_{\text {min }}$, whereas at least one of $\lambda$ will give positive value. From the result of $\lambda$ and $\xi_{\text {min }}$, first case is unstable and the last two cases are stable. Figure 8 illustrates the responses for unstable condition for all generators in Case 1.

In Figure $8(\mathrm{a})$, the response shows the angle deviation $\Delta \delta(t)$ of Generator 1 , Generator 2 and Generator 3 for Case 1 . From the response, it clearly shows that the response damping for all generators is increasing dramatically since the simulation starts. The same phenomenon can be seen in speed deviation $\Delta \omega(t)$ and torque deviation $\Delta T_{e}(t)$ for Case 1 as shown in Figure 8(b) and Figure 8(c), respectively. The damping increment of the response for all three figures indicates that Case 1 is an unstable case.

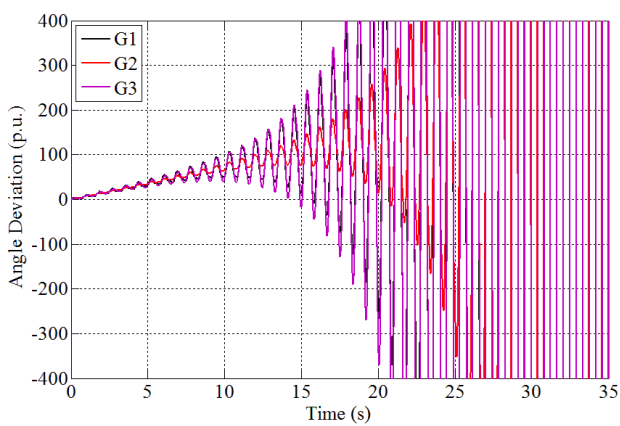

(a) Angle deviation

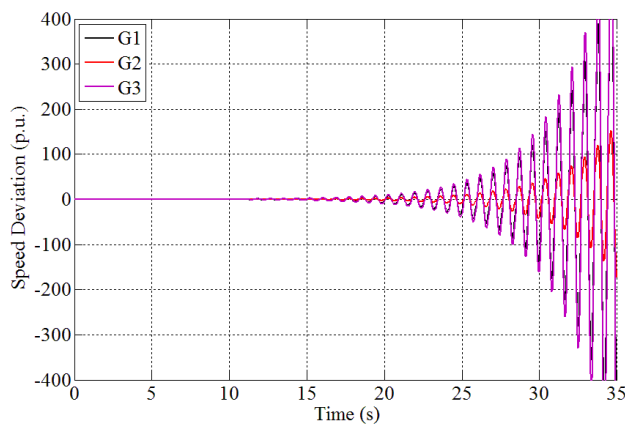

(b) Speed deviation

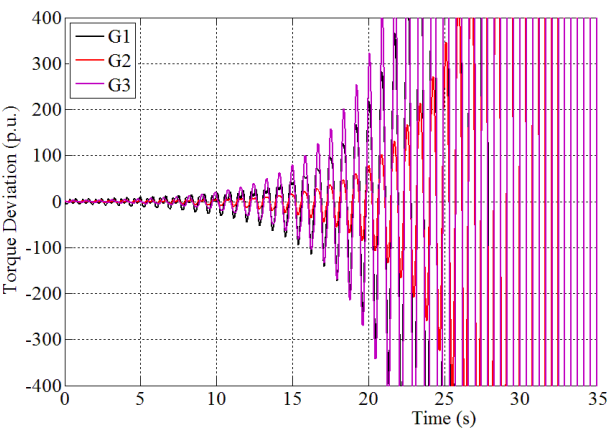

(c) Torque deviation

Figure 8. Responses for unstable condition for all generators in Case 1 
Table 3 tabulates the comparison of $K_{s}, K_{d}$, fitness $J$ and computation time for three different generators from EP, AIS, PSO and LS method for Case 1. In this study, no fitness value calculated for LS method as it is not an optimization technique. From Table III, PSO, EP, AIS and LS give negative values of $K_{s}$ and $K_{d}$ for first generator G1. For second and third generators, G2 and G3, all methods give positive $K_{s}$ and negative $K_{d}$. In this paper, value calculated with LS method was selected as a benchmark and all value calculated using EP, AIS and PSO estimation techniques were compared with this value. In Table III, the estimated value of $K_{s}$ and $K_{d}$ using EP, AIS and PSO showed similar values with the values estimated by LS. Among them, PSO gives the closest value compared to LS for all generators, followed by EP and finally AIS method. It shows that PSO technique manages to bring the most accurate value of $K_{s}$ and $K_{d}$ compared to EP and AIS. Since the values of $K_{s}$ and $K_{d}$ for all three generators are negative, this result indicates that all generators in Case 1 are unstable.

\begin{tabular}{|c|c|c|c|c|c|}
\hline Gen. & Tech & $K_{S}$ & $K_{d}$ & $J$ & Computation Time \\
\hline \multirow{4}{*}{1} & EP & -0.2563 & -3.2637 & 0.8901 & $18.0 \mathrm{~s}$ \\
\hline & AIS & -0.2451 & -4.9650 & 0.8561 & $7.5 \mathrm{~s}$ \\
\hline & PSO & -0.2769 & -2.4385 & 1.0000 & $20.8 \mathrm{~s}$ \\
\hline & LS & -0.2638 & -2.9425 & - & - \\
\hline \multirow{4}{*}{2} & EP & 2.3026 & -4.0787 & 0.8106 & $19.2 \mathrm{~s}$ \\
\hline & AIS & 2.5156 & -3.3527 & 0.8071 & $7.5 \mathrm{~s}$ \\
\hline & PSO & 2.1717 & -5.7955 & 1.0000 & $19.5 \mathrm{~s}$ \\
\hline & LS & 2.2533 & -5.0252 & - & - \\
\hline \multirow{4}{*}{3} & EP & 1.4169 & -1.963 & 0.8906 & $19.2 \mathrm{~s}$ \\
\hline & AIS & 1.3148 & -1.9171 & 0.8891 & $7.5 \mathrm{~s}$ \\
\hline & PSO & 1.4909 & -2.0073 & 1.0000 & $19.5 \mathrm{~s}$ \\
\hline & LS & 1.4572 & -1.9558 & 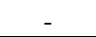 & - \\
\hline
\end{tabular}

From all methods, only PSO manage to achieve fitness value of 1.000 for all generators. The second highest of fitness value is EP, with range between $0.8506 \sim 0.8906$. AIS give the lowest value of fitness, with range between $0.8071 \sim 0.8891$. In terms of computation time, AIS is the fastest technique, manage to finish the optimization process in 7.5 seconds. PSO and EP are in the same level, able to finish the optimization process between 18 21 seconds. LS technique is not an optimization method, so no iteration value is recorded.

Table 4 shows the results of $\lambda$ and $\xi_{\min }$ for Case 1 . From eigenvalues $\lambda$ point of view, one of the eigenvalues is 0.0512 which is positive value. This confirms that Case 1 is an unstable case. Negative value of minimum damping ratio $\xi_{\min }$ was also verified the same conclusion.

Table 4. The results of Eigenvalues, Minimum Damping Ratio for Case 1

\begin{tabular}{cc}
\hline$\xi_{\min }$ & $\lambda$ \\
\hline \multirow{2}{*}{-1.0} & $0.1098 \pm \mathrm{j} 14.7919,0.2803 \pm \mathrm{j} 7.4378, \mathbf{0 . 0 5 1 2},-0.0003$, \\
& $-31.866,-26.8641 \pm \mathrm{j} 7.472,-24.1887 \pm \mathrm{j} 2.653,-17.892$. \\
\hline
\end{tabular}

Figure 9(a), Figure 9(b) and Figure 9(c) show the responses of $\Delta \delta(t), \Delta \omega(t)$ and $\Delta T_{e}(t)$ for Case 2, respectively. In Figure 9(a), the damping of angle deviation for all generators is decreasing gradually. When the simulation time reaches $50 \mathrm{~s}$, damping for G1, G2 and G3 are minimum but still not fully damped. Among three generators, G2 and G3 show almost the same responses. On the other hand, response of the first generator G1 is the most fluctuate. The same response patent can be seen in Figure 9(b) and Figure 9(c), which show speed deviation and torque deviation of Case 2, respectively. Same as Figure 9(a), the response of G1 is the most fluctuate compared to G2 and G3 for both Figure 9(b) and Figure 9(c). These results indicate that Case 2 is a stable case. 


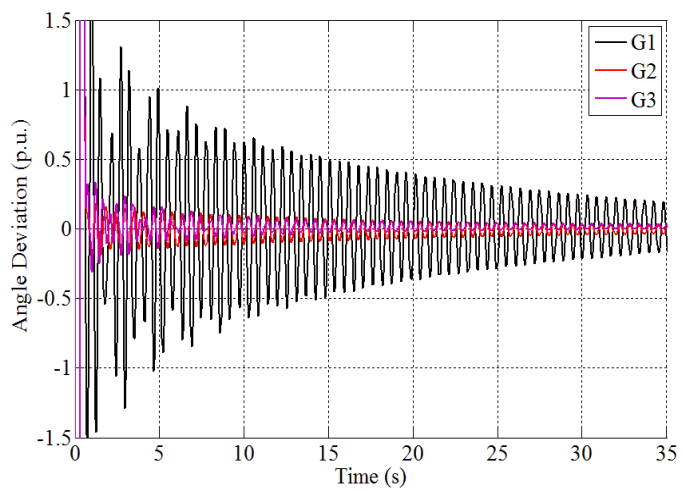

(a) Angle deviation

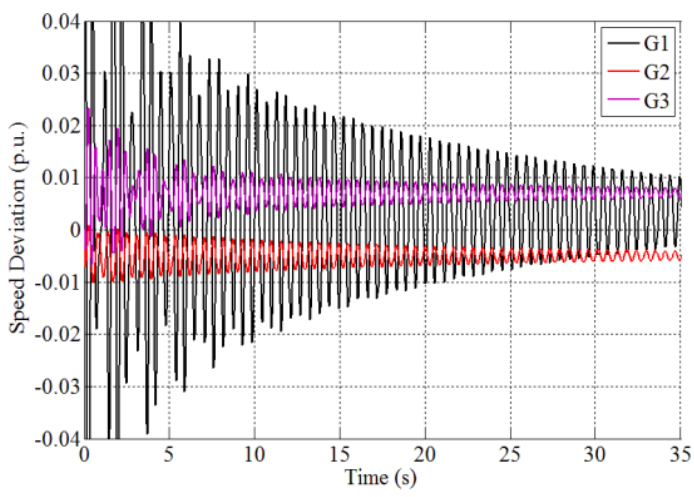

(b) Speed deviation

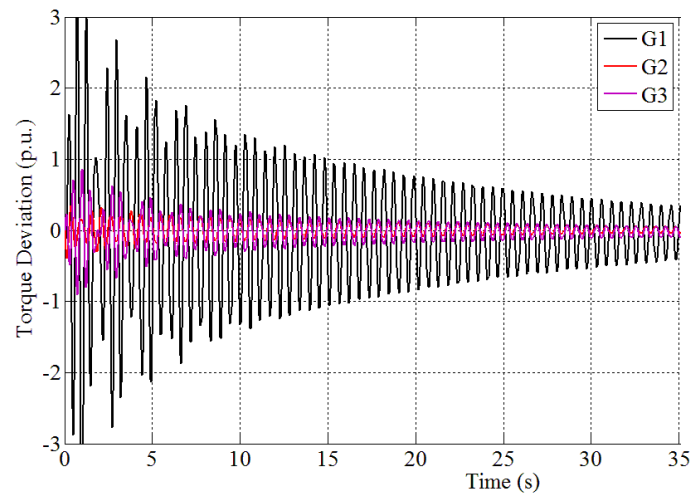

(c) Torque deviation

Figure 9. Responses for stable condition for all generators in Case 2

The value of torque coefficients $K_{s}$ and $K_{d}$ together with result of fitness $J$ and computation time for Case 2 are tabulated in Table 5. For Case 2, EP, AIS and PSO method give identical positive values of both $K_{s}$ and $K_{d}$, which verify that it is a stable case. Despite it is a stable case, LS method has demonstrated different results, a negative value of $K_{d}$ for generator G1 and negative value for both $K_{s}$ and $K_{d}$ for G3. This shows that LS technique failed to provide accurate results involving difficult cases such as Case 2 . The results of fitness $J$ and computation time shown in Table $\mathrm{V}$ are almost consistent with the previous Case 1 . PSO score 1.000 in fitness value for all generators. AIS is the fastest computation technique, which acquired 4.5 7.5 seconds to finish the optimize process. EP become the worst of all three method, with the slowest simulation takes 24 seconds to converge. Overall, PSO is the best technique to achieve the accurate assessment in accepted iteration limit.

Table 5. Comparisons of EP, AIS, PSO and LS method for Case 2

\begin{tabular}{cccccc}
\hline Gen. & Tech. & $K_{S}$ & $K_{d}$ & $J$ & Computation Time \\
\hline \multirow{6}{*}{1} & EP & 2.5026 & 3.0787 & 0.8106 & $19.2 \mathrm{~s}$ \\
& AIS & 2.5156 & 3.3527 & 0.8071 & $7.5 \mathrm{~s}$ \\
& PSO & 2.1717 & 2.7955 & 1.0000 & $19.5 \mathrm{~s}$ \\
& LS & 1.2090 & -0.7011 & - & - \\
& EP & 2.1959 & 6.9951 & 0.8695 & $24.0 \mathrm{~s}$ \\
2 & AIS & 2.2500 & 7.4038 & 0.8420 & $4.5 \mathrm{~s}$ \\
& PSO & 1.8114 & 6.5481 & 1.0000 & $19.5 \mathrm{~s}$ \\
& LS & 0.9002 & 4.5534 & - & - \\
& EP & 0.9973 & 0.0316 & 0.7870 & $20.4 \mathrm{~s}$ \\
3 & AIS & 0.9703 & 0.0103 & 0.7841 & $7.5 \mathrm{~s}$ \\
& PSO & 1.8537 & 0.0340 & 1.0000 & $18.2 \mathrm{~s}$ \\
& LS & -0.0012 & -0.0312 & - & - \\
\hline
\end{tabular}


Table 6 tabulates the value $\xi_{\min }$ and $\lambda$ for Case 2 . The value of $\xi_{\min }$ is 0.0065 which is nearly closed to negative value. For eigenvalues, although all values are negative, one of the $\lambda$ values is almost zero that is near to positive value. This shows that Case 2 is considered as stable case even though it is almost near to unstable region.

Table 6. The Results of Eigenvalues, Minimum Damping Ratio for Case 2

\begin{tabular}{cc}
\hline$\xi_{\min }$ & $\lambda$ \\
\hline \multirow{2}{*}{0.0065} & $-0.3103 \pm \mathrm{j} 14.348,-0.0730 \pm \mathrm{j} 11.282,-0.0012,-0.0001$, \\
& $-32.591,-30.772,-25.589 \pm \mathrm{j} 8.514,-18.474,-17.2905$. \\
\hline
\end{tabular}

The responses of $\Delta \delta(t), \Delta \omega(t)$ and $\Delta T_{e}(t)$ for Case 3 are shown respectively in Figure 10(a), Figure 10(b) and Figure 10(c). In Figure 10(a), the angle deviation for G1 is completely damped about $15 \mathrm{~s}$ after the simulation started. It is follows by $\Delta \delta(t)$ of G3 which stop fluctuate about $18 \mathrm{~s}$, and $\mathrm{G} 2$ at the $23^{\text {rd }}$ seconds after the simulation started. The speed deviation and torque deviation for Case 3 also show the same response patent, as shown in Figure 10(b) and Figure 10(c), respectively. Based on these three types of responses, Case 3 is classified as one of stable cases. More than that, Case 3 is damp faster than Case 2.

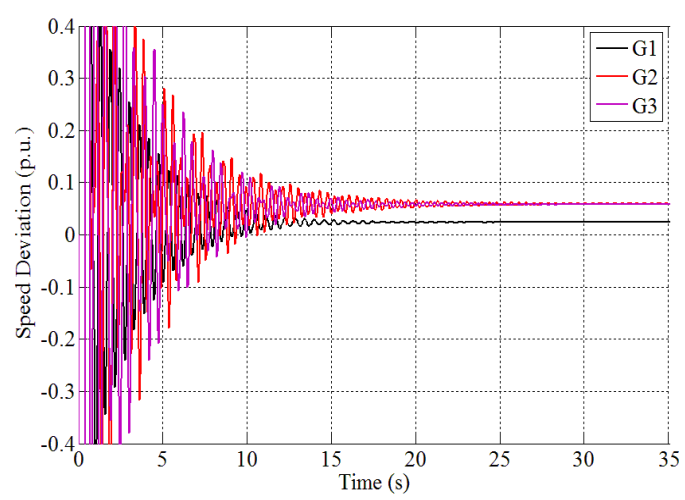

(a) Angle deviation

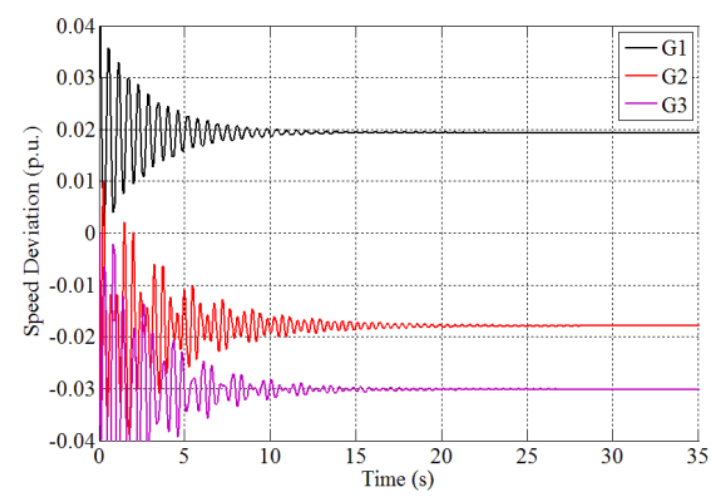

(b) Speed deviation

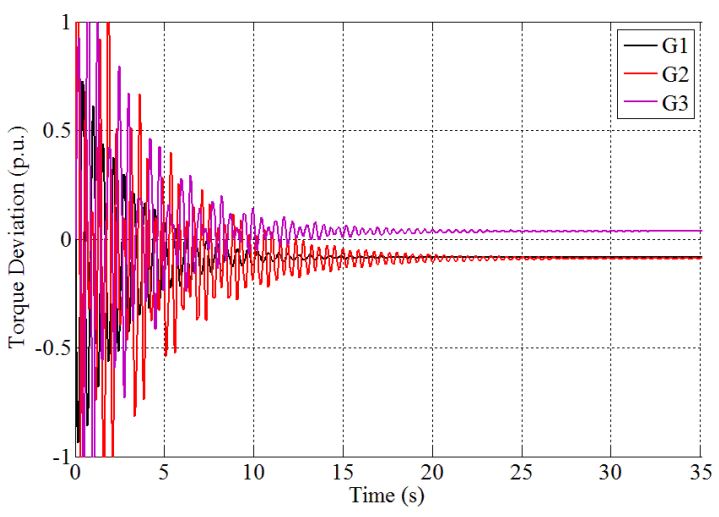

(c) Torque deviation

Figure 10. Responses for stable condition for all generators in Case 3

Table 7 shows the comparisons of $K_{s}, K_{d}$, fitness $J$ and computation time for Case 3. PSO, EP, AIS and LS estimation techniques give positive values of $K_{s}$ and $K_{d}$ for all generators G1, G2 and G3. This result indicates that Case 3 is stable case. In this case, PSO demonstrated the most identical value of $K_{s}$ and $K_{d}$ to LS method compared to the other two techniques. Also, from the result, PSO can calculate the fitness with 1.000 scores for all three generators. On the other hand, EP and AIS calculate the highest fitness for G2 with 
0.8534 and 0.8067 scores, respectively. Although AIS is the fastest optimization technique, the computation time for EP and PSO are still acceptable.

Table 7. Comparisons of EP, AIS, PSO and LS method for Case 3

\begin{tabular}{cccccc}
\hline Gen. & Tech. & $K_{S}$ & $K_{d}$ & $J$ & Computation Time \\
\hline \multirow{4}{*}{1} & EP & 6.7984 & 3.6458 & 0.8157 & $19.2 \mathrm{~s}$ \\
& AIS & 7.5737 & 3.8709 & 0.8051 & $4.5 \mathrm{~s}$ \\
& PSO & 5.1308 & 3.4876 & 1.0000 & $19.5 \mathrm{~s}$ \\
& LS & 4.0870 & 4.2304 & - & - \\
& EP & 5.1562 & 11.1108 & 0.8534 & $18.0 \mathrm{~s}$ \\
2 & AIS & 6.1249 & 14.9902 & 0.8067 & $7.5 \mathrm{~s}$ \\
& PSO & 4.4353 & 10.3909 & 1.0000 & $20.8 \mathrm{~s}$ \\
& LS & 2.2301 & 7.8529 & - & - \\
& EP & 2.6436 & 8.1310 & 0.7939 & $13.2 \mathrm{~s}$ \\
3 & IIS & 2.6122 & 8.0070 & 0.7871 & $7.5 \mathrm{~s}$ \\
& PSO & 2.8125 & 9.0231 & 1.0000 & $15.6 \mathrm{~s}$ \\
& LS & 1.0323 & 7.1655 & - & - \\
\hline
\end{tabular}

The result of $\lambda$ and $\xi_{\min }$ for Case 3 are showed in Table 8. Positive value of $\xi_{\min }$ and all negative value of eigenvalues, $\lambda$ verify the same conclusion.

Table 8. The results of Eigenvalues, Minimum Damping Ratio for Case 3

\begin{tabular}{cc}
\hline$\xi_{\min }$ & $\lambda$ \\
\hline \multirow{3}{*}{0.0499} & $-0.2026 \pm \mathrm{j} 14.3629,-0.3413 \pm \mathrm{j} 10.8919$, \\
& $-0.0001 \pm \mathrm{j} 0.0020,-32.5934,-30.3290$, \\
& $-25.5359 \pm \mathrm{j} 8.1913,-18.9565,-17.0365$. \\
\hline
\end{tabular}

\section{CONCLUSIONS}

This study has presented the capability of the proposed PSO technique in the assessment of angle stability, specifically in multi machine system. IEEE 9-bus test system is chosen for the study. PSO method is proven as the most reliable optimization technique compared to EP and AIS method, although all three techniques managed to calculate correctly all cases whether it is stable or unstable. Optimization by PSO has achieved the highest accuracy compared to the other two techniques. PSO produced the highest accuracy for all cases, compared to EP and AIS. From the iteration perspective, AIS recorded the fastest simulation time, while PSO and EP were almost the same. Despite this disadvantage, the time consumed for PSO simulation process is still minimal and acceptable.

\section{REFERENCES}

[1] P. Kundur, Power System Stability and Control, New York, U.S.A: McGraw-Hill Professional, 1994.

[2] J. Machowski, J.W. Bialek and J.R. Bumby, Power System Dynamics Stability and Control, United Kingdom: Wiley, 2008.

[3] M.F. El-Naggar and A.A. Shetaya, "Wide Area Angle Stability Assessment Tool Based on Statistics Standard Scope Technique", IEEE 16th International Conference on Environment and Electrical Engineering (EEEIC), 2016, pp. $1-6$.

[4] M. Oluic, M. Ghandhari and B. Berggren, "Methodology for Rotor Angle Transient Stability Assessment in Parameter Space", IEEE Transactions on Power System, Vol. 32, pp. 1202 - 1211, 2017.

[5] K. Seethalekshmi, S.N. Singh and S.C. Srivastava, "A Synchrophasor Assisted Frequency and Voltage Stability Based Load Shedding Scheme for Self-Healing of Power System”, IEEE Transactions on Smart Grid, Vol. 2, pp. $221-230,2011$.

[6] H. Johannsson, A.H. Nielsen and J. Ostergaard, "Wide-Area Assessment of Aperiodic Small Signal Rotor Angle Stability in Real-Time", IEEE Transactions on Power Systems, Vol. 28, pp. 4545 - 4557, 2013.

[7] E.A. Feilat, "Fast Estimation of Synchronizing and Damping Torque Coefficients Using an Adaptive Neural Network", in 42nd International Universities Power Engineering Conference, 2007, pp. 1049 - 1053.

[8] N.A.M. Kamari, I. Musirin and M.M. Othman, "EP Based Optimization for Estimating Synchronizing and Damping Torque Coefficients", Australian Journal of Basic and Applied Sciences, Vol. 4, pp. 3741-3754, 2010.

[9] S. Ekinci, H.L. Zeynelgil and A. Demiroren, "A Didactic Procedure for Transient Stability Simulation of a MultiMachine Power System Utilizing SIMULINK," International Journal of Electrical Engineering Education, Vol. 53(1), pp. $54-71,2016$.

[10] H.A.M. Moussa and Y.N. Yu, "Dynamic Interaction of Multi-Machine Power System and Excitation Control", IEEE Transactions on Power Apparatus and Systems, Vol. PAS-93, pp. 1150 - 1158, 1974. 
[11] M.H.M. Zaman, M.M. Mustafa and A. Hussain, "Critical Equivalent Series Resistance Estimation for Voltage Regulator Stability Using Hybrid System Identification and Neural Network", International Journal on Advanced Science, Engineering and Information Technology, Vol. 7, pp. 1381 - 1388, 2017.

[12] I. Moukhtar, A.A. Elbaset, A.Z.E. Dein, Y. Qudaih, E. Blagin, D. Uglanov and Y. Mitani, "A Developed Concentrated Solar Power Model using Artificial Neural Network Technique", Nineteenth International Middle East Power Systems Conference (MEPCON), 2017, pp. 1346 - 1351.

[13] J.A. Ali, M.A. Hannan and A. Mohamed, "Improved Indirect Field-Oriented Control of Induction Motor Drive Based PSO Algorithm", Jurnal Teknologi, Vol. 78, Issue 6-2, pp. 27 -32, 2016.

[14] M.K.M. Zamani, I. Musirin, S.I. Suliman, M.M. Othman and M.F.M. Kamal, "Multi-Area Economic Dispatch Performance Using Swarm Intelligence Technique Considering Voltage Stability", International Journal on Advanced Science, Engineering and Information Technology, Vol. 7, pp. 1 - 7, 2017.

[15] J.A. Ali, M.A. Hannan and A. Mohamed, "PSO algorithm for Three Phase Induction Motor drive with SVPWM Switching and V/f Control", IEEE International Conference on Power and Energy (PECon), 2014, pp. 250 - 254.

[16] B.W. Argo, Y. Hendrawan, D.F. Al-Riza and A.N.J. Laksono, "Optimization of PID Controller Parameters on Flow Rate Control System Using Multiple Effect Evaporator Particle Swarm Optimization", International Journal on Advanced Science, Engineering and Information Technology, Vol. 5, pp. 60 - 68, 2015.

[17] M.S. Hossain Lipu, M.A. Hannan, A. Hussain and M.H.M. Saad, "Optimal BP Neural Network Algorithm for State of Change Estimation of Lithium-Ion Battery Using PSO with PCA Feature Selection", Journal of Renewable and Sustainable Energy, Vol. 9, Issue 6, pp. 1 - 16, 2017.

[18] M. Basu, "Fast Convergence Evolutionary Programming for Economic Dispatch Problems", IET Generation, Transmission \& Distribution, Vol. 11, pp. 4009 - 4017, 2017.

[19] N.A. Salim and J. Maika, "Optimal Allocation of FACTS Device to Improve Voltage Profile and Power Loss using Evolutionary Programming Technique", IEEE Region 10 Conference (TENCON), 2016, pp. 1208 - 1215.

[20] S. Saravanan, S. Amosedinakaran, K. Karunanithi and N. Bhoopal, "Estimation of India's Energy Demand using Artificial Immune System", International Conference on Intelligent Computing and Control Systems (ICICCS), $2017,1150-1154$.

[21] A.A. Ibrahim, A. Mohamed, H. Shareef and S.P. Ghoshal, "Optimal Power Quality Monitor Placement in Power Systems Based on Particle Swarm Optimization and Artificial Immune System", 3rd Conference on Data Mining and Optimization (DMO), 2011, pp. $141-145$.

[22] M. Naserian, A. Karimi and S.E. Mirabdolahi, "Robust Optimal Controller Design for Induction Generator Driven by Variable-Speed Wind Turbine with STATCOM Using Immune Algorithm", International Journal on Advanced Science, Engineering and Information Technology, Vol. 1, pp. 476 - 480, 2011.

[23] S.R. Spea, "Economic-emission Dispatch Problem using Firefly Algorithm", Nineteenth International Middle East Power Systems Conference (MEPCON), 2017, pp. 671 - 766.

[24] L.A. Wong, H. Shareef, A. Mohamed and A.A. Ibrahim, "Novel Quantum-Inspired Firefly Algorithm for Optimal Quality Monitor Placement", Frontiers in Energy, Vol. 8, Issue 2, pp. 254 - 260, 2014. 